\title{
Anatomic fat depots and cardiovascular risk: a focus on the leg fat using nationwide surveys (KNHANES 2008-2011)
}

Eugene Han ${ }^{1,2,5}$, Yong-ho Lee ${ }^{1,2,3^{*}} \mathbb{0}$, Byung-Wan Lee ${ }^{1,2,3}$, Eun Seok Kang ${ }^{1,2,3}$, In-Kyu Lee ${ }^{4}$ and Bong-Soo Cha ${ }^{1,2,3}$

\begin{abstract}
Background: Although central fat is a well-known risk factor for cardiovascular disease (CVD) and cardiometabolic disorders, the effect of other regional fats or muscle distribution on CVD risk has not been fully investigated.

Methods: This was a cross-sectional study using nationally representative samples of 15,686 subjects from the 20082011 Korea National Health and Nutrition Examination Survey. Individual CVD risk was evaluated in adults aged $\geq 20$ without prior CVD, using atherosclerotic cardiovascular disease (ASCVD) risk equations according to the 2013 ACC/ AHA guidelines. Body composition was assessed by dual X-ray absorptiometry.

Results: Ratio of leg fat to total fat (LF/TF ratio) was the most predictive for CVD among body fat or muscle distribution parameters (AUC $=0.748,95 \% \mathrm{Cl} 0.741-0.755$ ). ASCVD risk score was gradually increased with decreased LF/ TF ratio $(P<0.001)$, and individuals whose LF/TF ratio in lowest tertile tended to belong to the high-risk (10-year risk $>10 \%)$ group compared to those in the highest tertile $(\mathrm{OR}=6.25,95 \% \mathrm{Cl}$ 5.60-6.98). Subjects in the lowest tertile showed increased risk of cardiometabolic risk factor components including obesity, hypertension, diabetes, dyslipidemia, chronic kidney disease, and albuminuria (OR range 2.57-11.24, all P < 0.001). In addition, a higher LF/TF ratio was associated with decreased ASCVD risk, even in subjects with multiple CVD risk factors. Multiple logistic regression analyses also demonstrated this association ( $\mathrm{OR}=1.85,95 \% \mathrm{Cl} 1.36-2.52)$.
\end{abstract}

Conclusions: Among various body composition parameters, LF/TF ratio was superior in predicting higher CVD risk and a higher LF/TF ratio was independently associated with decreased risk of CVD and each cardiometabolic risk factor.

Keywords: Cardiovascular disease risk factors, Risk score, Obesity, Metabolic syndrome

\section{Background}

As obesity has become an increasingly epidemic disease, the incidence of cardiovascular and metabolic diseases have risen to become public health issues. Overweight and obesity are related to cardiovascular, and all-cause mortality [1]. More specifically, it has been demonstrated that excessive visceral fat is related to an increased risk of hypertension [2] and diabetes [3]. Although the association between excessive abdominal fat and risk of

\footnotetext{
*Correspondence: yholee@yuhs.ac

${ }^{1}$ Division of Endocrinology, Department of Internal Medicine, Yonsei University College of Medicine, 50-1 Yonsei-ro, Seodaemun-gu, Seoul 03722, South Korea

Full list of author information is available at the end of the article
}

cardiovascular disease (CVD) and other cardiometabolic diseases has been established, investigation of a relationship between other body fat accumulation and cardiometabolic disease is limited. In addition, the different effect of regional body fat or muscle composition on CVD has not been fully understood.

Visceral adiposity is linked to altered myocardial glucose uptake [4], concentric left ventricular remodeling [5], and left atrial dysfunction [6]. In contrast, subcutaneous fat depot was associated with higher cardiac output and lower systemic vascular resistance [5]. Among subcutaneous fat, thigh girth or thigh fat is reported to attenuate risk for dyslipidemia [7] and glucose intolerance [8]. The protective effect of larger hip circumference 
to CVD has also been confirmed in previous studies of myocardial infarction [9], coronary heart disease (CHD) [10], and any CVD event [11]. Studies on lower-body fat have focused on the hip or thigh and indirectly assessed fat distribution by circumference. In addition, studies investigating on the effect of various body composition parameters on CVD risk are limited.

Dual-energy X-ray absorptiometry (DXA) has been recently used to assess body fat distribution as well as bone density. The regional fat measurement using DXA is reliable compared to computed tomography, a gold standard tool for measurement [12]. It also has the capability to scan whole body fat distribution with minimal radiation exposure.

We hypothesized that higher ratio of leg fat to total fat (LF/TF ratio) is related to low CVD risk regardless of other metabolic conditions. The aim of this study was to investigate the effect of each body fat or muscle distribution on CVD risk, and the association between LF/TF ratio and various CVD and cardiometabolic risk factor components in the general population.

\section{Methods}

\section{Study population}

This cross-sectional study utilized data collected from participants in the Korea National Health and Nutrition Examination Surveys (KNHANES) 2008-2011. This nationwide, population-based, annual survey is conducted by the Korean government to monitor public health [13]. In KNHANES data, medical history includes smoking habits, alcohol consumption, exercise level, and disease diagnosis and/or treatment, based on direct interviews and self-reporting. Each KNHANES is comprised of independent data sets from the general population. As described in Fig. 1, among 37,753 participants, we initially selected those aged $\geq 20$ without a prior history of CVD. We excluded those with missing data for DXA and CVD risk assessments, such that, 15,686 subjects (6761 men and 8925 women) were included in the analysis. All participants provided written informed consent to participate in the original KNHANES. The survey protocol was approved by the institutional review board of the Korean Centers for Disease Control and Prevention (2008-04EXP-01-C, 2009-07CON-03-2C, 2010-02CON21-C, and 2011-02CON-06C).

\section{Regional body distributions and cardiovascular disease risk}

Fat mass and muscle mass were measured using DXA (QDR 4800A; Hologic Inc., Bedford, MA, USA). The DXA scans provided soft tissue and bone measurements for both arms and both legs, and the trunk. Values for these regions include total mass, fat mass, muscle mass.

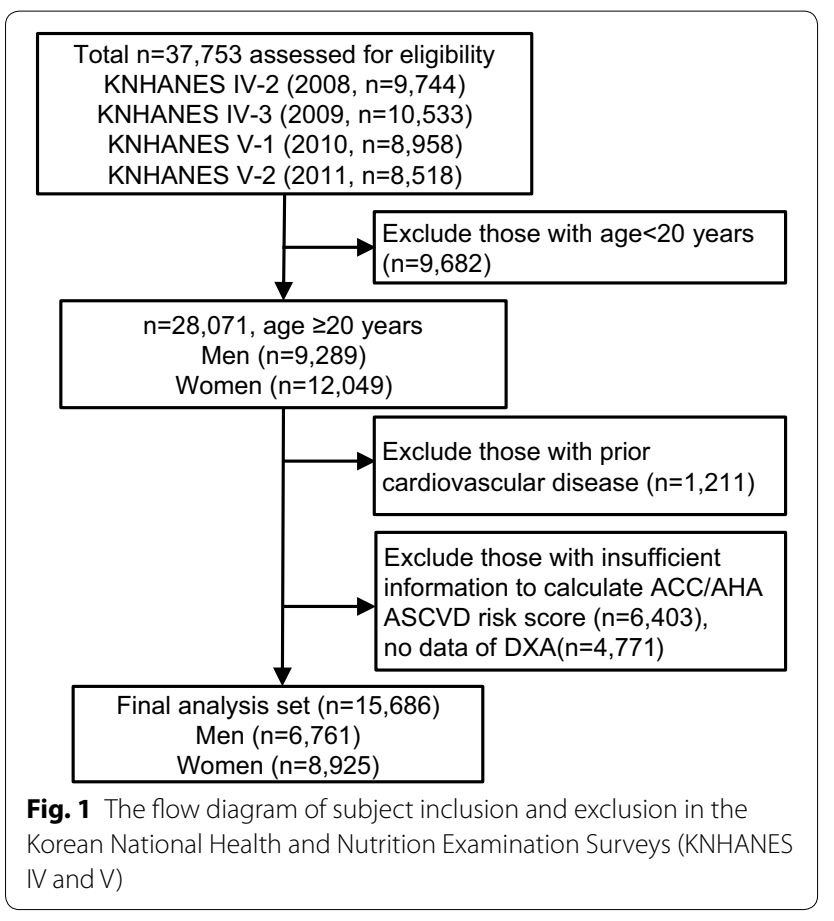

Leg measurement was assessed by sum of both legs including thigh and calf and arm measurement was indicated as sum of both side upper arms and forearms values. CVD risk was evaluated using various approaches, including the 10-year atherosclerotic cardiovascular disease (ASCVD) risk score from the 2013 American College of Cardiology/American Heart Association (ACC/AHA) guidelines [14]. In addition, the Framingham 10-year CVD risk score [15] and the Korean coronary heart disease (CHD) prediction model [16] were assessed. Leg muscle mass and each CVD risk score was analyzed and categorized into sex-specific tertiles. Moreover, LF/TF ratio was divided into tertiles according to obesity (body mass index $[\mathrm{BMI}] \geq 25 \mathrm{~kg} / \mathrm{m}^{2}$, Asianpacific criteria [17]) and sex. Individuals exhibiting ACC/ AHA ASCVD risk $>10 \%$, Framingham CVD risk $>20 \%$, and Korean CHD risk $>5 \%$ were classified into the highrisk group. We assessed regional body fat and muscle distributions' receiver operator characteristic (ROC) curve to predict and high CVD risk. In addition, calculations of the area under the ROC curve (AUC) were compared among the fat and muscle parameters.

\section{Cardiometabolic disease components}

During surveys, blood pressure was manually measured using mercury sphygmomanometers (Baumanometer; W.A. Baum, Copiague, NY) three times in resting, seated positions, and final blood pressure values were assessed by averaging the second and third blood pressure 
readings. Overnight ( $\geq 8-\mathrm{h}$ ) fasting blood and spot urine samples were collected, refrigerated, and transported to a central laboratory (NeoDin Medical Institute, Seoul, South Korea) within $24 \mathrm{~h}$. Diabetes was defined as using anti-diabetic agents, or who exhibited fasting plasma glucose $\geq 126 \mathrm{mg} / \mathrm{dL}$ or glycated hemoglobin $6.5 \%$. Individuals were considered to have hypertension if their systolic and/or diastolic blood pressure was $\geq 140 / 90 \mathrm{mmHg}$, or if they were taking anti-hypertensive medications. Central obesity was defined as waist circumference $\geq 90 \mathrm{~cm}$ in men and $\geq 85 \mathrm{~cm}$ in women [18]. Metabolic syndrome was based on the modified National Cholesterol Education Program Adult Treatment Panel III criteria, adopted for the Korean population [19]. Hyper low density lipoprotein (LDL) cholesterolemia was characterized as LDL $\geq 160 \mathrm{mg} / \mathrm{dL}$ for both sexes, and hypo high density lipoprotein (HDL) cholesterolemia was defined as HDL $<40 \mathrm{mg} / \mathrm{dL}$ for men, and $<50 \mathrm{mg} / \mathrm{dL}$ for women. Hypertriglyceridemia was defined as serum triglycerides $\geq 150 \mathrm{mg} / \mathrm{dL}$ or taking triglyceride lowering agents. Estimated glomerular filtration rate (eGFR) was calculated using the Chronic Kidney Disease Epidemiology collaboration equation [20], and chronic kidney disease was characterized as subjects with eGFR $<60 \mathrm{~mL} /$ $\min / 1.73 \mathrm{~m}^{2}$ [21]. Albuminuria was characterized as having more than one positive dip-stick test or urine albumin-to-creatinine ratio $>30 \mathrm{mg} / \mathrm{g}$ [21]. Excluding individuals with missing urine data, 819 subjects were categorized into sex-specific LF/TF ratio tertiles and analyzed for risk of albuminuria. Regular exercise was defined as "engaging in intense physical activity, that made one very tired or breathe much harder than usual, and involved more than $20 \mathrm{~min}$ per session at least 3 times per week" (e.g., running, jogging, mountain climbing, fast cycling, fast swimming, playing soccer, playing basketball, playing squash or singles tennis, transporting heavy objects, etc.). An age cutoff point to classify adults as younger or older was 50 years, the median value.

\section{Statistical analysis}

Data are presented as the mean \pm standard deviation for continuous variables, and as number, or percent for categorical variables. Simple correlation coefficients were used to assess correlations among regional fat depots. We analyzed participants' characteristics according to LF/TF ratio tertiles, using one-way analysis of variance (ANOVA) to compare continuous variables and, $x^{2}$ tests for categorical variables, followed by post hoc analyses using the Bonferroni method. To evaluate the association between LF/TF ratio and CVD risk, we attempted to minimize the effects of comorbidities. Subjects were classified according to the presence of hypertension, diabetes, metabolic syndrome, and obesity, and then $x^{2}$ tests were applied to each group. Multivariable adjusted logistic regression analyses were used to test the independent association between LF/TF ratio and high CVD risk (ACC/AHA ASCVD risk $>10 \%$, Framingham CVD risk $>20 \%$ or Korean CHD risk $>5 \%$ ) and between LF/ TF ratio and CVD risk factors. To measure the prediction accuracy of each body compositions for high CVD risk, we calculated and compared AUC of each regional fat parameters. The comparison of the AUC for ROC curves was performed on the basis of the Delong test [22]. As total cholesterol, triglyceride, HDL cholesterol, LDL cholesterol, insulin, and the homeostasis model assessment of insulin resistance (HOMA-IR) values were not normally distributed, analyses were performed using log-transformed data in order to achieve approximately symmetrical distributions. Statistical analyses were performed using IBM SPSS version 23.0 for Windows (IBM Corp., Armonk, NY, USA), and MedCalc (version 13.1; http://medcalc.software.informer.com/13.1); P 0.05 was considered statistically significant.

\section{Results}

Baseline characteristics by ratio of leg fat to total fat

A total of 15,686 individuals with no history of CVD were analyzed in the present study (Fig. 1). Individuals with a higher LF/TF ratio tended to have smaller waist circumference, lower blood pressure, and more favorable metabolic conditions (glucose, insulin, HOMA-IR, LDL cholesterol, triglyceride, HDL cholesterol, and eGFR) (Table 1). Subsequently, a low prevalence of hypertension, diabetes, metabolic syndrome and chronic kidney disease was observed in the highest LF/TF ratio tertile in both non-obese and obese population. The correlation between leg fat and arm fat was strong (Pearson coefficient $=0.808, \mathrm{P}<0.001$ ), while the association between leg fat and trunk fat was relatively weak (Pearson coefficient $=0.647, \mathrm{P}<0.001)$ in our population.

\section{Various fat depots and cardiovascular disease risk}

Leg fat was superior to arm, total, or trunk fat distributions in predicting higher values of ACC/AHA ASCVD risk scores (Fig. 2a). In addition, the LF/TF ratio (AUC $=0.748,95 \% \mathrm{CI} 0.741-0.755$ ) showed greater accuracy in predicting high ACC/AHA ASCVD risk compared to other fat distributions $\left(\mathrm{AUC}_{\mathrm{arm} \text { fat } /}\right.$ total fat ratio $=0.568,95 \% \mathrm{CI} 0.561-0.576, \mathrm{AUC}_{\text {trunk fat } /}$ total fat ratio $=0.717,95 \% \mathrm{CI} 0.709-0.724, \mathrm{AUC}_{\text {waist cir- }}$ cumference $=0.659,95 \%$ CI 0.652-0.667) (Fig. 2b). After stratifying into sex-specific leg fat and total fat tertile separately, individuals with a higher leg fat even in higher total fat had shown lower ACC/AHA ASCVD risk compared to lower leg fat and lower total fat groups (Fig. 2c). LF/TF ratio tertiles were well-correlated with 
Table 1 Baseline characteristics of study population by categories of ratio of leg fat to total fat (LF/TF ratio)

\begin{tabular}{|c|c|c|c|c|c|c|}
\hline & \multicolumn{6}{|c|}{ Tertiles of LF/TF ratio } \\
\hline & \multicolumn{3}{|l|}{ Lean } & \multicolumn{3}{|l|}{ Obese } \\
\hline & $\begin{array}{l}\text { Lowest tertile } \\
(\mathrm{n}=3592)\end{array}$ & $\begin{array}{l}\text { Second tertile } \\
(n=3590)\end{array}$ & $\begin{array}{l}\text { Highest tertile } \\
(\mathrm{n}=3593)\end{array}$ & $\begin{array}{l}\text { Lowest tertile } \\
(n=1631)\end{array}$ & $\begin{array}{l}\text { Second tertile } \\
(n=1627)\end{array}$ & $\begin{array}{l}\text { Highest tertile } \\
(n=1633)\end{array}$ \\
\hline Leg fat (kg) & $4.3 \pm 1.4$ & $5.1 \pm 1.7^{\S}$ & $5.4 \pm 1.8^{\S, \|}$ & $5.1 \pm 1.4$ & $6.4 \pm 1.7^{\S}$ & $7.6 \pm 2.1^{\S, \|}$ \\
\hline Total fat (kg) & $15.9 \pm 4.1$ & $15.6 \pm 4.4^{\S}$ & $13.9 \pm 4.0^{\xi, \|}$ & $21.5 \pm 4.9$ & $22.6 \pm 5.3^{\S}$ & $23.0 \pm 5.4^{\S}$ \\
\hline LF/TF ratio (\%) & $26.4 \pm 3.2$ & $32.5 \pm 3.3^{5}$ & $38.7 \pm 4.8^{\S, \|}$ & $23.8 \pm 2.2$ & $28.1 \pm 1.8^{\S}$ & $33.0 \pm 3.2^{\S, \|}$ \\
\hline Age (year) & $57.0 \pm 13.5$ & $47.5 \pm 15.0^{\S}$ & $38.2 \pm 14.2^{\S, \|}$ & $55.7 \pm 12.3$ & $51.1 \pm 13.9^{\S}$ & $43.6 \pm 14.6^{5, \|}$ \\
\hline Men (\%) & 40.4 & 40.3 & 40.3 & 49.2 & 49.2 & 49.2 \\
\hline $\begin{array}{l}\text { Waist circumference } \\
(\mathrm{cm})\end{array}$ & $80.4 \pm 6.5$ & $76.7 \pm 6.9^{\S}$ & $72.5 \pm 7.1^{\S, \|}$ & $92.0 \pm 6.8$ & $91.0 \pm 7.0^{\S}$ & $88.6 \pm 7.3^{\S, \|}$ \\
\hline $\mathrm{BMI}\left(\mathrm{kg} / \mathrm{m}^{2}\right)$ & $22.7 \pm 1.7$ & $22.0 \pm 1.9^{\S}$ & $20.9 \pm 2.1^{\S, \|}$ & $27.4 \pm 2.1$ & $27.5 \pm 2.3$ & $27.2 \pm 2.2^{11}$ \\
\hline $\begin{array}{l}\text { Systolic blood pres- } \\
\text { sure }(\mathrm{mmHg})\end{array}$ & $122.1 \pm 17.9$ & $114.0 \pm 16.6^{5}$ & $107.8 \pm 13.6^{\S, \|}$ & $125.9 \pm 16.3$ & $122.8 \pm 16.4^{\S}$ & $117.4 \pm 15.4^{\S, \|}$ \\
\hline $\begin{array}{l}\text { Diastolic blood pres- } \\
\text { sure }(\mathrm{mmHg})\end{array}$ & $76.2 \pm 10.4$ & $73.1 \pm 10.2^{\S}$ & $70.1 \pm 9.3^{5, \|}$ & $79.9 \pm 10.2$ & $78.9 \pm 10.4^{\S}$ & $76.5 \pm 10.2^{\S, \|}$ \\
\hline $\begin{array}{l}\text { Fasting plasma } \\
\text { glucose }(\mathrm{mg} / \mathrm{dL})\end{array}$ & $102.1 \pm 26.6$ & $93.6 \pm 19.3^{\S}$ & $89.3 \pm 12.2^{\S, \|}$ & $111.0 \pm 31.8$ & $101.6 \pm 21.0^{\S}$ & $95.2 \pm 15.9^{\S, \|}$ \\
\hline $\begin{array}{l}\text { Total cholesterol } \\
(\mathrm{mg} / \mathrm{dL})^{\mathrm{a}}\end{array}$ & $194.4 \pm 36.6$ & $185.2 \pm 33.2^{\S}$ & $172.9 \pm 30.0^{\S, \|}$ & $200.3 \pm 37.4$ & $198.3 \pm 36.5$ & $191.3 \pm 35.3^{\S, \|}$ \\
\hline $\begin{array}{l}\text { HDL cholesterol } \\
(\mathrm{mg} / \mathrm{dL})^{\mathrm{a}}\end{array}$ & $50.7 \pm 12.0$ & $54.6 \pm 13.0^{5}$ & $57.5 \pm 12.6^{5, \|}$ & $47.3 \pm 10.8$ & $47.7 \pm 11.1$ & $49.8 \pm 11.0^{\S, \|}$ \\
\hline $\begin{array}{l}\text { Triglycerides (mg/ } \\
\mathrm{dL})^{\mathrm{a}}\end{array}$ & $154.4 \pm 114.1$ & $115.6 \pm 87.4^{\S}$ & $85.1 \pm 67.8^{\S, \|}$ & $190.6 \pm 150.1$ & $174.0 \pm 121.3^{\S}$ & $136.3 \pm 98.3^{\S, \| 1}$ \\
\hline $\begin{array}{l}\text { LDL cholesterol } \\
(\mathrm{mg} / \mathrm{dL})^{\mathrm{a}}\end{array}$ & $118.4 \pm 33.5$ & $112.5 \pm 29.5^{\S}$ & $102.8 \pm 26.6^{\S, \|}$ & $122.1 \pm 34.0$ & $122.0 \pm 32.5$ & $119.6 \pm 31.3$ \\
\hline $\begin{array}{l}\mathrm{eGFR}(\mathrm{mL} / \\
\left.\mathrm{min} / 1.73 \mathrm{~m}^{2}\right)\end{array}$ & $90.1 \pm 16.1$ & $97.3 \pm 16.5^{\S}$ & $103.6 \pm 16.1^{\S, \|}$ & $98.1 \pm 16.8$ & $92.2 \pm 16.2^{\S}$ & $97.9 \pm 16.6^{5, \|}$ \\
\hline WBC $\left(10^{3} / \mu \mathrm{L}\right)$ & $6.1 \pm 1.7$ & $5.8 \pm 1.6^{\S}$ & $5.6 \pm 1.5^{5, \|}$ & $6.6 \pm 1.8$ & $6.5 \pm 1.7$ & $6.2 \pm 1.6^{5, \| 1}$ \\
\hline Insulin $(y \mathrm{U} / \mathrm{mL})^{\mathrm{a}}$ & $9.4 \pm 5.4$ & $8.7 \pm 3.9^{\S}$ & $8.4 \pm 3.4^{\S, \|}$ & $13.0 \pm 7.6$ & $12.1 \pm 5.8$ & $11.1 \pm 4.6$ \\
\hline $\mathrm{HOMA}-\mathrm{R}^{\mathrm{a}}$ & $2.4 \pm 2.3$ & $2.0 \pm 1.1^{\S}$ & $1.9 \pm 0.8^{\S, \|}$ & $3.7 \pm 3.4$ & $3.1 \pm 1.9^{\S}$ & $2.6 \pm 1.2^{\S, \|}$ \\
\hline Alcohol (g/week) & $73.8 \pm 122.5$ & $63.5 \pm 113.3^{\S}$ & $44.6 \pm 84.2^{\S, \|}$ & $82.0 \pm 132.0$ & $78.9 \pm 123.5$ & $67.8 \pm 113.4^{\S}$ \\
\hline Current smoker (\%) & 20.5 & 21.6 & 20.9 & 21.3 & 23.3 & 24.2 \\
\hline $\begin{array}{l}\text { Regular exercise } \\
(\%)^{b}\end{array}$ & 24.2 & 24.7 & $22.0^{\S}$ & 26.4 & 27.2 & 28.5 \\
\hline Hypertension (\%) & 37.5 & $17.9^{\S}$ & $7.0^{5, \|}$ & 53.8 & $40.7^{\S}$ & $24.0^{\S, \| 1}$ \\
\hline Diabetes (\%) & 15.2 & $4.1^{\S}$ & $1.1^{\S, \|}$ & 26.4 & $12.2^{\S}$ & $4.3^{\S, \|}$ \\
\hline $\begin{array}{l}\text { Metabolic syndrome } \\
(\%)\end{array}$ & 37.6 & $11.9^{\S}$ & $2.4^{\S, \|}$ & 72.4 & $60.2^{\S}$ & $35.0^{\S, \|}$ \\
\hline $\begin{array}{l}\text { Chronic kidney } \\
\text { disease }(\%)^{c}\end{array}$ & 4.3 & $2.1^{\S}$ & $0.6^{\xi, \|}$ & 5.3 & 3.7 & $2.2^{\S}$ \\
\hline
\end{tabular}

Data for continuous variables were expressed as mean \pm standard deviation, and categorical variables were expressed as percent (\%)

$B M I$ body mass index; $H D L$ cholesterol, high density lipoprotein cholesterol; $L D L$ cholesterol, low density lipoprotein cholesterol; eGFR estimated glomerular filtration rate; WBC white blood cell; HOMA-IR homeostasis model assessment of insulin resistance

$\S \mathrm{P}<0.05$ by post hoc analyses when compared with lowest tertile

$\| \mathrm{P}<0.05$ by post hoc analyses when compared with second tertile

a Log-transformed

b Regular exercise was defined as engaging in intense physical activity, that made one very tired or breathe much harder than usual, and involved more than 20 min per session at least 3 times per week

c Chronic kidney disease was defined if subjects had an eGFR less than $60 \mathrm{ml} / \mathrm{min} / 1.73 \mathrm{~m}^{2}$ 

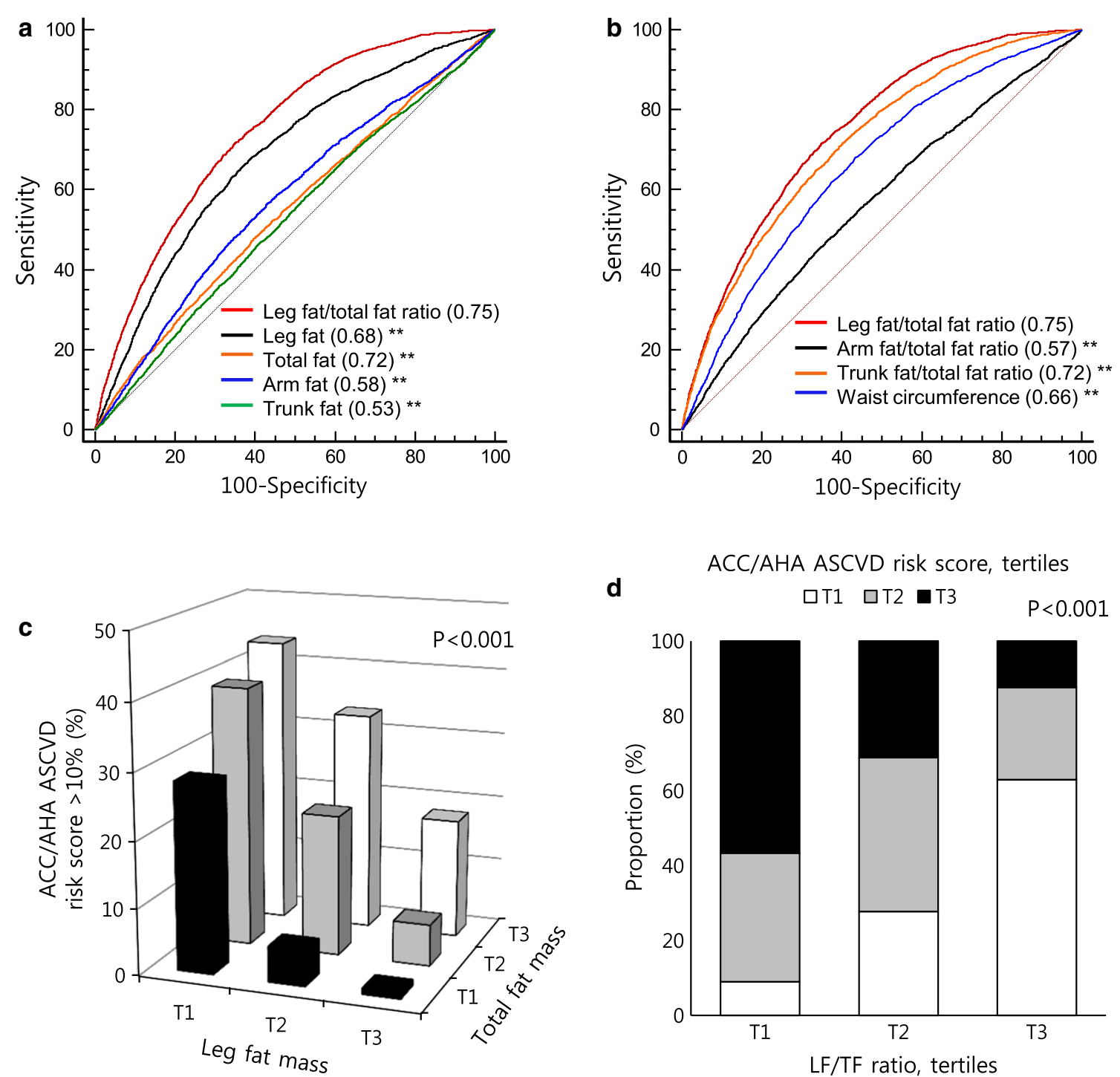

Fig. 2 Regional body fat distributions, receiver operating characteristic (ROC) curves, and high ACC/AHA ASCVD risk. a ROC curves to predict high ACC/AHA ASCVD risk (>10\%) for each regional fat. b ROC curves to predict high ACC/AHA ASCVD risk for other regional fat ratios and waist circumference. $\mathbf{c}$ Proportion of individuals with high ACC/AHA ASCVD risk by leg fat and total fat tertiles. $\mathbf{d}$ Proportion of individuals with ACC/AHA ASCVD risk score tertiles by LF/TF ratio. ${ }^{* *} P<0.001$

ACC/AHA ASCVD score tertiles (Fig. 2d). The other risk scores showed similar results (Additional file 1: Figure S1).

\section{Ratio of leg fat to total fat and cardiometabolic disease risk components}

We have demonstrated a relationship between LF/TF ratio and CVD risk; likewise, we analyzed the risks of each cardiometabolic factor component as related to LF/TF ratio. In Table 2, individuals with the lowest LF/ TF ratio tertile had the highest risk of obesity, hypertension, diabetes, dyslipidemia, metabolic syndrome, and kidney dysfunction (OR range 2.64-11.24, all $\mathrm{P}<0.001)$. To assess the association between LF/TF ratio and CVD risk independent of other covariates, we performed multiple logistic regression analysis. After sequential adjustment for confounding covariates such as age, sex, obesity, smoking, alcohol, systolic blood pressure, serum glucose, dyslipidemia, BMI, menopause state and kidney function, individuals with lower LF/TF ratios were found to have significantly higher adjusted odds ratios (AORs) for high ACC/AHA ASCVD risk $(\mathrm{AOR}=1.85,95 \% \mathrm{CI} 1.36-2.52, \mathrm{P}<0.001)$ compared to subjects with the highest LF/TF ratio. The AOR for high 
Table 2 Odds ratio and $95 \%$ confidential interval of cardiometabolic disease risk factors and high CVD risk according to sex-specific LF/TF ratio tertiles in adults

\begin{tabular}{|c|c|c|c|}
\hline & \multicolumn{3}{|l|}{ LF/TF ratio } \\
\hline & Highest tertile & Second tertile & Lowest tertile \\
\hline \multicolumn{4}{|l|}{ Risk factor } \\
\hline Obesity ${ }^{\mathrm{a}}$ & 1 (referent) & $3.72(3.33-4.15)$ & $7.67(6.81-8.23)$ \\
\hline Central obesity $^{\mathrm{b}}$ & 1 (referent) & $4.29(3.83-4.82)$ & $9.22(8.16-10.42)$ \\
\hline Hypertension & 1 (referent) & $2.05(1.80-2.34)$ & $3.57(3.14-4.07)$ \\
\hline Diabetes & 1 (referent) & $2.88(2.19-3.78)$ & $8.89(6.87-11.50)$ \\
\hline $\begin{array}{l}\text { Hyper LDL-cho- } \\
\text { lesterolemiac }\end{array}$ & 1 (referent) & $2.14(1.76-2.60)$ & $2.72(2.23-3.32)$ \\
\hline $\begin{array}{l}\text { Hypo HDL-cho- } \\
\text { lesterolemia }^{d}\end{array}$ & 1 (referent) & $1.92(1.76-2.11)$ & $2.91(2.64-3.22)$ \\
\hline $\begin{array}{l}\text { Hypertriglyceri- } \\
\text { demia }^{\mathrm{e}}\end{array}$ & 1 (referent) & $3.01(2.69-3.37)$ & $6.14(5.46-6.90)$ \\
\hline $\begin{array}{l}\text { Metabolic } \\
\text { syndrome }\end{array}$ & 1 (referent) & $4.06(3.53-4.67)$ & $11.24(9.78-12.93)$ \\
\hline $\begin{array}{l}\text { Chronic kidney } \\
\text { disease }^{f}\end{array}$ & 1 (referent) & $1.97(1.30-2.99)$ & $2.57(1.73-3.81)$ \\
\hline Albuminuriag & 1 (referent) & $2.03(1.21-3.41)$ & $2.64(1.57-4.44)$ \\
\hline \multicolumn{4}{|c|}{ High ACC/AHA ASCVD risk } \\
\hline Crude & 1 (referent) & $2.53(2.25-2.84)$ & $6.25(5.60-6.98)$ \\
\hline Model 1 & 1 (referent) & $1.95(1.56-2.44)$ & $3.69(2.98-4.57)$ \\
\hline Model 2 & 1 (referent) & $1.65(1.30-2.08)$ & $2.91(2.31-3.66)$ \\
\hline Model 3 & 1 (referent) & $1.26(0.92-1.71)$ & $1.85(1.36-2.52)$ \\
\hline \multicolumn{4}{|c|}{ High Framingham CVD risk } \\
\hline Crude & 1 (referent) & $2.48(2.16-2.84)$ & $6.32(5.56-7.18)$ \\
\hline Model 1 & 1 (referent) & $1.96(1.61-2.39)$ & $4.09(3.39-4.94)$ \\
\hline Model 2 & 1 (referent) & $1.70(1.38-2.09)$ & $3.21(2.63-3.92)$ \\
\hline Model 3 & 1 (referent) & $1.43(1.07-1.91)$ & $2.37(1.79-3.15)$ \\
\hline \multicolumn{4}{|c|}{ High Korean CHD risk } \\
\hline Crude & 1 (referent) & $3.43(2.30-5.13)$ & $7.98(5.48-11.63)$ \\
\hline Model 1 & 1 (referent) & $2.47(1.59-3.82)$ & $4.08(2.69-6.17)$ \\
\hline Model 2 & 1 (referent) & $2.39(1.52-3.75)$ & $3.64(2.37-5.60)$ \\
\hline Model 3 & 1 (referent) & $1.94(1.11-3.38)$ & $2.57(1.52-4.37)$ \\
\hline
\end{tabular}

Risk factors are adjusted for age, sex, exercise, smoking, and alcohol drinking Model 1: adjusted for age, and sex

Model 2: adjusted for age, sex, exercise, smoking, alcohol drink, and BMI Model 3: adjusted for age, sex, exercise, smoking, alcohol drink, BMI, systolic blood pressure, fasting blood glucose, hyper LDL-cholesterolemia, eGFR, and menopause

a Obesity was defined $\mathrm{BMI} \geq 25 \mathrm{~kg} / \mathrm{m}^{2}$

b Central obesity was defined waist circumference $\geq 90 \mathrm{~cm}$ in men, $\geq 85 \mathrm{~cm}$ in women

c Hyper LDL-cholesterolemia was characterized as calculated LDL cholesterol $\geq 160 \mathrm{mg} / \mathrm{dL}$

d Hypo HDL-cholesterolemia was defined as serum HDL cholesterol $<40 \mathrm{mg} / \mathrm{dL}$ for men, and $<50 \mathrm{mg} / \mathrm{dL}$ for women

e Hypertriglyceridemia was defined as serum triglycerides $\geq 150 \mathrm{mg} / \mathrm{dL}$ or taking triglyceride lowering agents

f Chronic kidney disease was defined if subjects had an eGFR less than $60 \mathrm{~mL} /$ $\min / 1.73 \mathrm{~m}^{2}$

g Albuminuria was characterized urine albumin-creatinine ratio $>30 \mathrm{mg} / \mathrm{g}$ or urine protein $\geq 2$ positive. The LF/TF ratio tertile was newly categorized in 819 subjects with urine test results risk of CVD gradually increased in lower LF/TF ratio, in other models.

Ratio of leg fat to total fat is associated with cardiovascular disease risks independent of hypertension, diabetes, and metabolic syndrome

The increasing average ACC/AHA ASCVD risk scores was linked with decreased LF/TF ratio in both non-obese and obese group, and this finding was shown in both young and old age group (Additional file 2: Figure S2). The distribution of high-risk subjects according to LF/ TF ratio tertiles showed the same trend as ACC/AHA ASCVD risk scores regardless of age group $(9.1,20.1$, and $38.4 \%$ for highest, second, and lowest tertiles, respectively, $\mathrm{P}<0.001$ ).

Subgroup analyses were conducted to identify the LF/ TF ratio and CVD risk independent of other metabolic conditions. Figure 3 depicts that, regardless of the presence of hypertension, diabetes, metabolic syndrome or insulin resistance state, a higher LF/TF ratio was associated with lower ACC/AHA ASCVD risks in both non-obese and obese group. When other CVD risk assessments were applied, similar results were observed (Additional file 3: Figure S3, Additional file 4: Figure S4). Furthermore, people with a higher LF/TF ratio were less likely to have multiple CVD risk factors. In individuals with a higher LF/TF ratio having multiple risk factors, their CVD risk was not as high as those with lower LF/ TF ratios, regardless of CVD risk models (Fig. 3e). We further assessed ACC/AHA ASCVD risk in leg muscle mass in relation to LF/TF ratio. Along with a higher LF/TF ratio, higher leg muscle mass was associated with lower ACC/AHA ASCVD risk and both LF/TF ratio and leg muscle mass synergistically decreased ACC/AHA ASCVD risk (Fig. 3f).

\section{Discussion}

This large, nationally representative, population-based study demonstrated that LF/TF ratio was superior to other parameters in predicting high CVD risk, and that people with a higher LF/TF ratio tended to have decreased risk of CVD and other cardiometabolic risk components. LF/TF ratio was associated with CVD risk independent of hypertension, diabetes, obesity, and metabolic syndrome, and more risk attenuation was observed in healthy conditions. In addition, subjects with a higher LF/TF ratio and multiple CVD risk factors had a CVD risk comparable to those with a lower LF/TF ratio and few CVD risk factors. The association between LF/ TF ratio and CVD risk remained significant after adjusting for other confounding factors.

Regional fat accumulation, rather than BMI, has a strong correlation with cardiometabolic disease 

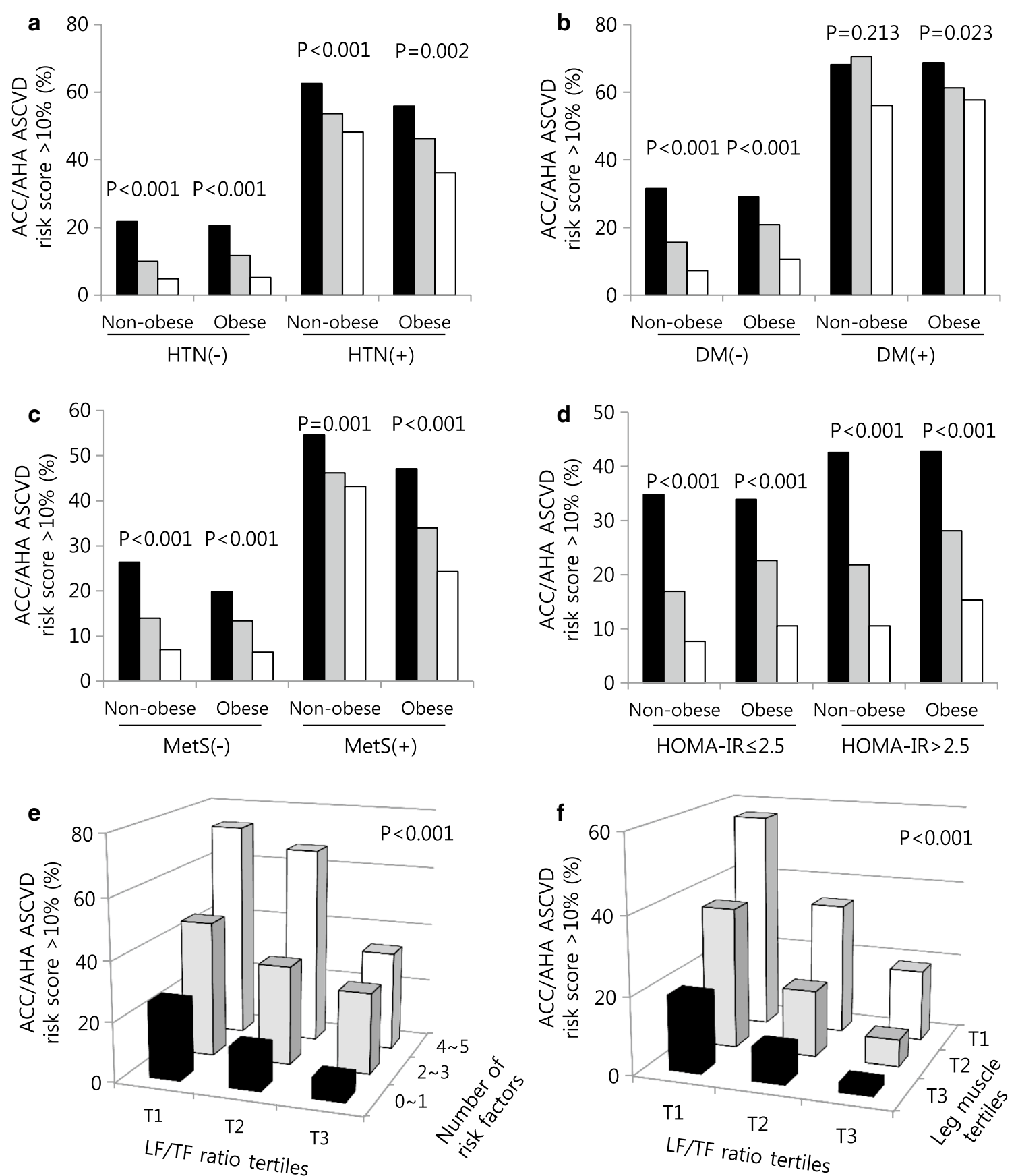

Fig. 3 Difference in ACC/AHA ASCVD risk according to LF/TF ratio tertiles, subgroup analysis. Proportion of individuals with high ACC/AHA ASCVD risk (>10\%) stratified by a hypertension, $\mathbf{b}$ diabetes, $\mathbf{c}$ metabolic syndrome, $\mathbf{d}$ insulin resistance (HOMA-IR), and e central obesity. f Number of cardiovascular risk factors according to LF/TF tertiles. Risk factors are obesity, hypertension, diabetes, hyper LDL-cholesterolemia, and hypertriglyceridemia. HTN hypertension, DM diabetes mellitus, MetS metabolic syndrome, HOMA-IR homeostasis model assessment of insulin resistance

incidence [10]. The role of each body fat depot to metabolic health seems to vary. Abdominal fat accumulation with visceral fat is adversely correlated with cardiometabolic disease [3, 23-25], accounted for disparate phenotype of subcutaneous and visceral adipocyte [26]. High visceral fat with low subcutaneous fat could be a marker for fat accumulation in vessel and liver, accelerating atherosclerosis and fatty liver $[27,28]$. Furthermore, the role of subcutaneous fat seems to be diverse according to the body compartment. Deep subcutaneous adipose tissue 
was demonstrated to have similar phenotype of visceral fat, with increased insulin resistance and high CVD risk score, whereas superficial subcutaneous adipose tissue was the opposite [29]. Subcutaneous fat in the lowerbody region has more potent properties of metabolism [30-32]. In healthy postmenopausal women, leg fat mass was a significant independent predictor of insulin sensitivity, reducing risk for hyperinsulinemia and insulin resistance, whereas trunk fat was associated with increased risk of those conditions [30]. In addition, this association was consistently maintained independent of body weight [31]. The results of our study support those of previous studies regarding the importance of body fat distribution as a predisposing risk factor for CVD and the extent to which LF/TF ratio is a better predictor compared to upper-body fat (ratio of arm fat to total fat) or trunk fat. Furthermore, we found that a high LF/TF ratio had stronger association with lower CVD risk when combined with sufficient leg muscle mass.

Previous studies using DXA provided limited results on the effect of regional adiposity distributions on CVD risk and were mainly confined to postmenopausal women. A study of postmenopausal women showed the association between regional fat and indirect CVD risk using hyperinsulinemia, and dyslipidemia [31]. Since then, a Danish group reports that peripheral fat mass correlated with aortic calcification in elderly females [33]. Both reports emphasized that localization of fat mass was more important than total body weight to atherosclerosis. The correlation between regional fat distribution on cardiometabolic risk factors, including blood pressure, HOMAIR, high-sensitivity C-reactive protein, and dyslipidemia has been recently reported in a European study [30]. However, it too was limited to postmenopausal women and lacked of a comprehensive CVD risk assessment. To our knowledge, the present study is the first investigate on the impact of leg fat distribution on comprehensive CVD risk in a large general Korean population.

Although the biologic mechanism of each body fat depot has not been fully investigated, the different regional fat deposit seems to have different functional properties. Subcutaneous fat in the gluteofemoral region is considered a 'metabolic sink' due to its low rate of lipolysis, fatty acid uptake and blood flow [34]. In terms of adipose tissue lipolysis, the leg fat is the most sensitive adipose tissue to insulin [35]. These distinct features of lower-body fat prevent chronic exposure to high lipid concentration. In addition, adiponectin level and insulin sensitivity correlates with leg fat mass [32, 36]. Another study demonstrated the profound secretion of lipokine, palmitoleate from leg fat using venoarterial difference sampling [37]. A lower LF/TF ratio was associated with increased risk of each cardiometabolic risk factor component in our results. It was more closely related to the risk of obesity and diabetes than to hypertension and dyslipidemia, with the highest OR associated with metabolic syndrome, suggesting that a lower LF/TF ratio combined with another cardiometabolic risk factor component might synergistically increase its harmful effect. Furthermore, a lower LF/TF ratio was related to chronic kidney disease and even albuminuria risk in our results. Considering albuminuria as a marker of a premature stage of inflammation and CVD progression [38], a lower-body fat depot might be involved the in early development of CVD. As an interesting finding, individuals with multiple risk components and a higher LF/TF ratio had a CVD risk equivalent to those with a lower LF/TF ratio and few risk factors. The current study not only complemented the previous studies by showing a negative association of LF/TF ratio with CVD risk, but also revealed that higher LF/TF ratio is related with attenuated CVD risk even in high risk group. Moreover, the proportion at high risk was the highest in non-obese individuals in the lowest LF/TF ratio tertile, highlighting the importance of the LF/TF ratio in non-obese patients. These findings support previous studies suggesting the importance of the fat distribution effect to risk of CVD in people with normal BMI [39], and implying that regional fat can distinguish high CVD risk patients in a non-obese group [40, 41]. In non-obese group with diabetes, the correlation was not significant in our result might suggest the overwhelmed impact of diabetes on CVD.

To converse or attenuate the inappropriate impact of visceral fat, exercise should be recommended. 12-week standard aerobic exercise program effectively reduced visceral adipose tissue, and change in visceral fat area, but not subcutaneous fat was significantly associated with insulin resistance [42]. 1-year exercise and lifestyle modification program decreased visceral fat, but not subcutaneous fat, which resulted in enhancing adiponectin levels [43]. This impact of exercise was not limited in adult, but also was shown in obese adolescent individuals [44]. In addition, a multidisciplinary clinical trial including CTscan, metabolomics, and transcriptomics suggested that promoting subcutaneous fat expansion could be a therapeutic option for reducing metabolic complications in obese individuals impending diabetes or cardiovascular diseases [45]. Restoring dysregulated inflammatory and oxidative phosphorylation pathways in the adipose tissue would be a promising target for the treatment of atherosclerosis and insulin resistance [46].

This study has some limitations. First, due to its crosssectional design, we could not assert direct causality between LF/TF ratio and CVD risk. In addition, we applied indirect parameters to assess CVD risk. However, we used multiple surrogate CVD risk models with 
detailed statistical analyses to overcome this issue. Second, KHNASE did not collect each individual's medication information, and the potential medication effect on CVD risk was not analyzed. Third, DXA could not exactly distinguish between subcutaneous fat and intramuscular fat.

Despite these limitations, our study has several strengths. First, it utilized data from a large, national, population-based study, guaranteeing statistical reliability without selection bias. We were able to divide sex-specific LF/TF ratio tertiles, whereas previous studies on lower-body fat were conducted mostly in women. Second, various equations and adjustment models were applied and they consistently showed similar results. Third, we limited the study population by excluding those with prior CVD history, to prevent reverse causality. The novelty of our results was that LT/TF ratio was superior to other regional fat depots as a predictive marker of high CVD risk. Furthermore, CVD risk lowering impact of $\mathrm{LF} / \mathrm{TF}$ ratio was more prominent among individuals with high CVD risks.

The LF/TF ratio may distinguish individuals with a high CVD risk and provide reinforced risk modification to those subjects. In addition, our finding that a higher LF/ TF ratio might have a beneficial effect on CVD risk, could be a clue for "obesity paradox" which refers the decreased risk of death in overweight individuals compared to those in normal weight. Although we only analyzed adult population in this study using DXA, technical assessment of body composition [47], and association between regional fat depots and cardiometabolic risk in obese adolescents needs to be further investigated. Prospective, welldesigned, longitudinal studies with sufficient laboratory and cardiovascular imaging resources are warranted to identify the complex relationship between LF/TF ratio and CVD risk.

\section{Conclusions}

This nationwide survey of a representative sample of Korean population demonstrated that LF/TF ratio was important for predicting higher CVD risk and, was associated with increased risks of CVD, independent for other cardiovascular factors.

\section{Additional files}

Additional file 1: Figure S1. Proportion of individuals with CVD risk score tertiles by LF/TF ratio.

Additional file 2: Figure S2. Difference in ACC/AHA ASCVD risk according to LF/TF ratio tertiles. ACC/AHA ASCVD risk stratified by $\mathbf{A}$ obesity, and B age. Proportion of individuals with high ACC/AHA ASCVD risk (>10\%) stratified by cobesity, and $\mathbf{d}$ age.
Additional file 3: Figure S3. Difference in Framingham CVD risk according to LF/TF ratio tertiles, subgroup analysis. Proportion of individuals with high CVD risk (>20\%) stratified by $\mathbf{A}$ hypertension, B diabetes, C metabolic syndrome, and D insulin resistance (HOMA-IR). E Number of cardiovascular risk factors according to LF/TF tertiles. Risk factors are obesity, hypertension, diabetes, hyper LDL-cholesterolemia, and hypertriglyceridemia.

Additional file 4: Figure S4. Difference in Korean CHD risk according to LF/TF ratio tertiles, subgroup analysis. Proportion of individuals with high CHD risk ( $>5 \%$ ) stratified by $\mathbf{A}$ hypertension, B diabetes, $\mathbf{C}$ metabolic syndrome, and $\mathbf{D}$ insulin resistance (HOMA-IR). E Number of cardiovascular risk factors according to LF/TF tertiles. Risk factors are obesity, hypertension, diabetes, hyper LDL-cholesterolemia, and hypertriglyceridemia.

\section{Abbreviations}

ACC/AHA: American College of Cardiology/American Heart Association; ANOVA: analysis of variance; ASCVD: atherosclerotic cardiovasuclar disease; BMI: body mass index; CHD: coronary heart disease; $\mathrm{Cl}$ : confidence interval; CVD: cardiovascular disease; DXA: dual-energy X-ray absorptiometry; eGFR: estimated glomerular filtration rate; HDL: high density lipoprotein; HOMA-IR: homeostasis model assessment of insulin resistance; hs-CRP: high-sensitivity C-reactive protein; KNHANES: Korea National Health and Nutrition Examination Surveys; LDL: low density lipoprotein; LF/TF ratio: leg fat to total fat ratio; OR: odds ratio.

\section{Authors' contributions}

EH and YL contributed to study design, data collection, data analyses, and writing of the report. BWL, ESK, IKL, and BSC were responsible for overall supervision and contributed to data analyses and writing of the report. All authors had access to the data, commented on the report drafts. All authors read and approved the final manuscript.

\section{Author details \\ ${ }^{1}$ Division of Endocrinology, Department of Internal Medicine, Yonsei Univer- sity College of Medicine, 50-1 Yonsei-ro, Seodaemun-gu, Seoul 03722, South Korea. ${ }^{2}$ Graduate School, Yonsei University College of Medicine, Seoul, South Korea. ${ }^{3}$ Institue of Endocrine Research, Yonsei University College of Medicine, Seoul, South Korea. ${ }^{4}$ Division of Endocrinology, Department of Internal Medi- cine, Kyungpook National University School of Medicine, Daegu, South Korea. ${ }^{5}$ Division of Endocrinology, Department of Internal Medicine, Keimyung University School of Medicine, Daegu, South Korea.}

\section{Acknowledgements}

The authors are very grateful to officers who conducted KNHANES.

\section{Competing interests}

The authors declare that they have no competing interests.

\section{Availability of data and materials}

All data files are derived from the Division of Chronic Disease Surveillance of the Korea Centers for Disease Control and Prevention in the Ministry of Health and Welfare database, http://knhanes.cdc.go.kr/knhanes/eng/index.do, which is publically available to researchers who agree to conform to ethical research principles.

\section{Consent for publication}

If the manuscript is accepted, we approve it for publication in Cardiovascular Diabetology.

\section{Ethics approval and consent to participate}

All participants provided written informed consent to participate in the original KNHANES. The survey protocol was approved by the institutional review board of the Korean Centers for Disease Control and Prevention (2008-04EXP01-C, 2009-07CON-03-2C, 2010-02CON-21-C, and 2011-02CON-06C).

\section{Funding}

This research was supported by the grant from the Korea Healthcare Technology Research \& Development (R\&D) Project through the Korea Health Industry 
Development Institute (KHIDI), funded by the Ministry of Health and Welfare, Republic of Korea (HI14C2476 and HI16C1501).

\section{Publisher's Note}

Springer Nature remains neutral with regard to jurisdictional claims in published maps and institutional affiliations.

Received: 8 February 2017 Accepted: 18 April 2017

Published online: 26 April 2017

\section{References}

1. Twig G, Yaniv G, Levine H, Leiba A, Goldberger N, Derazne E, Ben-Ami Shor D, Tzur D, Afek A, Shamiss A, et al. Body-mass index in 2.3 million adolescents and cardiovascular death in adulthood. N Engl J Med. 2016;374(25):2430-40.

2. Hayashi T, Boyko EJ, Leonetti DL, McNeely MJ, Newell-Morris L, Kahn SE, Fujimoto WY. Visceral adiposity and the prevalence of hypertension in Japanese Americans. Circulation. 2003;108(14):1718-23.

3. Meisinger $C$, Doring $A$, Thorand $B$, Heier M, Lowel $H$. Body fat distribution and risk of type 2 diabetes in the general population: are there differences between men and women? The MONICA/KORA Augsburg cohort study. Am J Clin Nutr. 2006:84(3):483-9.

4. Kim G, Jo K, Kim KJ, Lee YH, Han E, Yoon HJ, Wang HJ, Kang ES, Yun M. Visceral adiposity is associated with altered myocardial glucose uptake measured by (18)FDG-PET in 346 subjects with normal glucose tolerance, prediabetes, and type 2 diabetes. Cardiovasc Diabetol. 2015;14:148.

5. Neeland IJ, Gupta S, Ayers CR, Turer AT, Rame JE, Das SR, Berry JD, Khera A, McGuire DK, Vega GL, et al. Relation of regional fat distribution to left ventricular structure and function. Circ Cardiovasc Imaging. 2013;6(5):800-7.

6. Evin M, Broadhouse KM, Callaghan FM, McGrath RT, Glastras S, Kozor R, Hocking SL, Lamy J, Redheuil A, Kachenoura N, et al. Impact of obesity and epicardial fat on early left atrial dysfunction assessed by cardiac MRI strain analysis. Cardiovasc Diabetol. 2016;15(1):164.

7. Terry RB, Stefanick ML, Haskell WL, Wood PD. Contributions of regional adipose tissue depots to plasma lipoprotein concentrations in overweight men and women: possible protective effects of thigh fat. Metab, Clin Exp. 1991;40(7):733-40.

8. Snijder MB, Visser M, Dekker JM, Goodpaster BH, Harris TB, Kritchevsky SB, De Rekeneire N, Kanaya AM, Newman AB, Tylavsky FA, et al. Low subcutaneous thigh fat is a risk factor for unfavourable glucose and lipid levels, independently of high abdominal fat. The Health ABC Study. Diabetologia. 2005;48(2):301-8.

9. Yusuf S, Hawken S, Ounpuu S, Bautista L, Franzosi MG, Commerford P, Lang CC, Rumboldt Z, Onen CL, Lisheng L, et al. Obesity and the risk of myocardial infarction in 27,000 participants from 52 countries: a casecontrol study. Lancet. 2005;366(9497):1640-9.

10. Canoy D, Boekholdt SM, Wareham N, Luben R, Welch A, Bingham S, Buchan I, Day N, Khaw KT. Body fat distribution and risk of coronary heart disease in men and women in the European prospective investigation into cancer and nutrition in norfolk cohort: a population-based prospective study. Circulation. 2007;116(25):2933-43.

11. de Koning L, Merchant AT, Pogue J, Anand SS. Waist circumference and waist-to-hip ratio as predictors of cardiovascular events: meta-regression analysis of prospective studies. Eur Heart J. 2007;28(7):850-6.

12. Glickman SG, Marn CS, Supiano MA, Dengel DR. Validity and reliability of dual-energy $\mathrm{X}$-ray absorptiometry for the assessment of abdominal adiposity. J Appl Physiol. 2004;97(2):509-14.

13. Lee YH, Kim SU, Song K, Park JY, Kim do Y, Ahn SH, Lee BW, Kang ES, Cha BS, Han KH. Sarcopenia is associated with significant liver fibrosis independently of obesity and insulin resistance in nonalcoholic fatty liver disease: nationwide surveys (KNHANES 2008-2011). Hepatology. 2016;63(3):776-86.

14. Goff DC Jr, Lloyd-Jones DM, Bennett G, Coady S, D'Agostino RB, Gibbons R, Greenland P, Lackland DT, Levy D, O'Donnell CJ, et al. 2013 ACC/ AHA guideline on the assessment of cardiovascular risk: a report of the American College of Cardiology/American Heart Association Task Force on Practice Guidelines. Circulation. 2014;129(25 Suppl 2):S49-73.
15. D’Agostino RB Sr, Vasan RS, Pencina MJ, Wolf PA, Cobain M, Massaro JM, Kannel WB. General cardiovascular risk profile for use in primary care: the Framingham Heart Study. Circulation. 2008;117(6):743-53.

16. Jee SH, Jang Y, Oh DJ, Oh BH, Lee SH, Park SW, Seung KB, Mok Y, Jung KJ, Kimm H, et al. A coronary heart disease prediction model: the Korean Heart Study. BMJ Open. 2014;4(5):e005025.

17. Song do K, Hong YS, Lee H, Oh JY, Sung YA, Kim Y. Increased epicardial adipose tissue thickness in type 2 diabetes mellitus and obesity. Diabetes Metab J. 2015;39(5):405-13.

18. Yoon YS, Oh SW. Optimal waist circumference cutoff values for the diagnosis of abdominal obesity in korean adults. Endocrinol Metab. 2014;29(4):418-26.

19. Son JW, Park CY, Kim S, Lee HK, Lee YS, Insulin Resistance as Primary Pathogenesis in Newly Diagnosed DNTDPiKSG. Changing clinical characteristics according to insulin resistance and insulin secretion in newly diagnosed type 2 diabetic patients in Korea. Diabetes Metab J. 2015;39(5):387-94.

20. Levey AS, Stevens LA, Schmid CH, Zhang YL, Castro AF 3rd, Feldman HI, Kusek JW, Eggers P, Van Lente F, Greene T, et al. A new equation to estimate glomerular filtration rate. Ann Intern Med. 2009;150(9):604-12.

21. National Kidney F. K/DOQI clinical practice guidelines for chronic kidney disease: evaluation, classification, and stratification. Am J Kidney Dis Off J Natl Kidney Found. 2002;39(2 Suppl 1):S1-266.

22. DeLong ER, DeLong DM, Clarke-Pearson DL. Comparing the areas under two or more correlated receiver operating characteristic curves: a nonparametric approach. Biometrics. 1988;44(3):837-45.

23. Larsson B, Svardsudd K, Welin L, Wilhelmsen L, Bjorntorp P, Tibblin G. Abdominal adipose tissue distribution, obesity, and risk of cardiovascular disease and death: 13 year follow up of participants in the study of men born in 1913. Br Med J. 1984;288(6428):1401-4.

24. Al-Daghri NM, Al-Attas OS, Wani K, Alnaami AM, Sabico S, Al-Ajlan A, Chrousos GP, Alokail MS. Sensitivity of various adiposity indices in identifying cardiometabolic diseases in Arab adults. Cardiovasc Diabetol. 2015;14:101.

25. Peng $Y$, Wang $H$, Chen F, Huang FY, Xia TL, Liao YB, Chai H, Wang PJ, Zuo $Z L$, Liu W, et al. The influence of body composition on renal function in patients with coronary artery disease and its prognostic significance: a retrospective cohort study. Cardiovasc Diabetol. 2016;15(1):106.

26. Wajchenberg BL. Subcutaneous and visceral adipose tissue: their relation to the metabolic syndrome. Endocr Rev. 2000;21(6):697-738.

27. Bouchi R, Takeuchi T, Akihisa M, Ohara N, Nakano Y, Nishitani R, Murakami M, Fukuda T, Fujita M, Minami l, et al. High visceral fat with low subcutaneous fat accumulation as a determinant of atherosclerosis in patients with type 2 diabetes. Cardiovasc Diabetol. 2015;14:136.

28. Bouchi R, Nakano Y, Ohara N, Takeuchi T, Murakami M, Asakawa M, Sasahara Y, Numasawa M, Minami I, Izumiyama H, et al. Clinical relevance of dual-energy X-ray absorptiometry (DXA) as a simultaneous evaluation of fatty liver disease and atherosclerosis in patients with type 2 diabetes. Cardiovasc Diabetol. 2016;15:64.

29. Marinou K, Hodson L, Vasan SK, Fielding BA, Banerjee R, Brismar K, Koutsilieris M, Clark A, Neville MJ, Karpe F. Structural and functional properties of deep abdominal subcutaneous adipose tissue explain its association with insulin resistance and cardiovascular risk in men. Diabetes Care. 2014;37(3):821-9.

30. Peppa M, Koliaki C, Hadjidakis DI, Garoflos E, Papaefstathiou A, Katsilambros N, Raptis SA, Dimitriadis GD. Regional fat distribution and cardiometabolic risk in healthy postmenopausal women. Eur J Intern Med. 2013;24(8):824-31.

31. Van Pelt RE, Evans EM, Schechtman KB, Ehsani AA, Kohrt WM. Contributions of total and regional fat mass to risk for cardiovascular disease in older women. Am J Physiol Endocrinol Metab. 2002;282(5):E1023-8.

32. Goss AM, Gower BA. Insulin sensitivity is associated with thigh adipose tissue distribution in healthy postmenopausal women. Metab Clin Exp. 2012;61(12):1817-23.

33. Tanko LB, Bagger $Y Z$, Alexandersen P, Larsen PJ, Christiansen C. Peripheral adiposity exhibits an independent dominant antiatherogenic effect in elderly women. Circulation. 2003;107(12):1626-31.

34. Manolopoulos KN, Karpe F, Frayn KN. Gluteofemoral body fat as a determinant of metabolic health. Int J Obes. 2010;34(6):949-59.

35. Meek SE, Nair KS, Jensen MD. Insulin regulation of regional free fatty acid metabolism. Diabetes. 1999;48(1):10-4. 
36. Snijder MB, Flyvbjerg A, Stehouwer CD, Frystyk J, Henry RM, Seidell JC, Heine RJ, Dekker JM. Relationship of adiposity with arterial stiffness as mediated by adiponectin in older men and women: the Hoorn Study. Eur J Endocrinol Eur Feder Endocr Soc. 2009;160(3):387-95.

37. Pinnick KE, Neville MJ, Fielding BA, Frayn KN, Karpe F, Hodson L. Gluteofemoral adipose tissue plays a major role in production of the lipokine palmitoleate in humans. Diabetes. 2012;61(6):1399-403.

38. de Zeeuw D, Parving HH, Henning RH. Microalbuminuria as an early marker for cardiovascular disease. J Am Soc Nephrol JASN. 2006:17(8):2100-5.

39. Kim S, Kyung C, Park JS, Lee SP, Kim HK, Ahn CW, Kim KR, Kang S. Normalweight obesity is associated with increased risk of subclinical atherosclerosis. Cardiovasc Diabetol. 2015;14:58.

40. Park SJ, Yang HM, Seo KW, Choi SY, Choi BJ, Yoon MH, Hwang GS, Tahk SJ, Sheen SS, Choi BI, et al. The relationship between coronary atherosclerosis and body fat distribution measured using dual energy $X$-ray absorptiometry. Atherosclerosis. 2016;248:190-5.

41. Fu X, Zhu F, Zhao X, Ma X, Zhu S. Central fat accumulation associated with metabolic risks beyond total fat in normal BMI Chinese adults. Ann Nutr Metab. 2014;64(2):93-100.
42. Lesser IA, Guenette JA, Hoogbruin A, Mackey DC, Singer J, Gasevic D, Lear SA. Association between exercise-induced change in body composition and change in cardiometabolic risk factors in postmenopausal South Asian women. Appl Physiol Nutr Metab. 2016;41(9):931-7.

43. Borel AL, Nazare JA, Smith J, Almeras N, Tremblay A, Bergeron J, Poirier P, Despres JP. Visceral and not subcutaneous abdominal adiposity reduction drives the benefits of a 1-year lifestyle modification program. Obesity (Silver Spring). 2012;20(6):1223-33.

44. Staiano AE, Marker AM, Beyl RA, Hsia DS, Katzmarzyk PT, Newton RL. A randomized controlled trial of dance exergaming for exercise training in overweight and obese adolescent girls. Pediatr Obes. 2017;12(2):120-8.

45. Torres-Perez E, Valero M, Garcia-Rodriguez B, Gonzalez-Irazabal Y, Calmarza P, Calvo-Ruata L, Ortega C, Garcia-Sobreviela MP, Sanz-Paris A, Artigas JM, et al. The FAT expandability (FATe) project: biomarkers to determine the limit of expansion and the complications of obesity. Cardiovasc Diabetol. 2015:14:40.

46. Moreno-Viedma V, Amor M, Sarabi A, Bilban M, Staffler G, Zeyda M, Stulnig TM. Common dysregulated pathways in obese adipose tissue and atherosclerosis. Cardiovasc Diabetol. 2016;15(1):120.

47. Horan M, Gibney E, Molloy E, McAuliffe F. Methodologies to assess paediatric adiposity. Ir J Med Sci. 2015;184(1):53-68.

\section{Submit your next manuscript to BioMed Central and we will help you at every step:}

- We accept pre-submission inquiries

- Our selector tool helps you to find the most relevant journal

- We provide round the clock customer support

- Convenient online submission

- Thorough peer review

- Inclusion in PubMed and all major indexing services

- Maximum visibility for your research

Submit your manuscript at www.biomedcentral.com/submit 